\title{
The Ultra-stable Scanning Transmission Electron Holography Microscope (STEHM)
}

\author{
Rodney A. Herring ${ }^{1}$ and David Hoyle ${ }^{2}$ \\ 1. CAMTEC, MENG, University of Victoria, British Columbia V8W 2Y2 Canada \\ 2. Hitachi High-Technology, Ltd., 89 Rexdale Blvd., Toronto, M9W 6A4 Canada
}

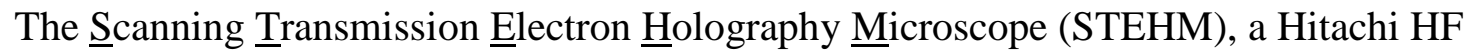
3300v equipped with the first Cs + coma TEM aberration corrector, i.e., an aplanatic TEM referred to as the b-corr, and the first Cs + Cc STEM aberration corrector referred to as the sccorr has been installed at the University of Victoria, Canada. The STEHM is an ultra-high resolution, ultra-stable electron microscope maintaining excellent atomic resolution stability for recording times of $120 \mathrm{~s}$, Fig. 1, an unprecedented length of time, maintaining spatial frequencies of $70 \mathrm{pm}$ within its lattice images. Other than the inherent stability of its electron optical system that can produce $\sim 35 \mathrm{pm}$ Fourier intensities for optimal imaging conditions, the residual stability of the STEHM is achieved by having an environmentally stable laboratory, as reported earlier [1], and a structurally stable specimen as reported in this abstract where a stable specimen for measuring the spatial resolution of the electron microscope has not yet been found. This is readily seen in Fig. 1 in the change in the crystal orientation of gold nanoparticles, now well known, during electron beam observation results in a change in contrast of the lattice fringes where some fringes recorded for $120 \mathrm{~s}$ are stronger in contrast than the $2 \mathrm{~s}$ image. The changes in the Au crystal's structure are verifiable in their Fourier transforms by the appearance and disappearance of Fourier peak intensities. Within the amorphous carbon substrate, specimen instabilities also exist. Although it is very difficult to follow the change in the amorphous structure by imaging, its periodic structures are manifested in its diffraction pattern consisting of speckle intensities. Self-interference of the speckle intensities can be used to follow the stability of the amorphous structure by using either a wavefront splitting method or an amplitude splitting method $[2,3]$. The amplitude splitting method involves self-interfering each speckle intensity, which is carried by the direct beam and the gold crystal's Bragg diffracted beams, using an electron biprism as described fully elsewhere [4]. Monitoring the contrast of the a-carbon's interference fringes, Fig. 2, enables the stability of the a-carbon structure to be followed during electron beam irradiation. This interference method also enables the absolute phase of the amorphous specimen to be measured necessary for determining its structure [3, 4]. Fig. 2 shows the change in fringe contrast within the amorphous speckle intensities due to its changing structure, which causes decoherence of the speckle intensities. Thus, both the gold nanocrystals and its a-carbon support film, which are commonly used for measuring the spatial resolution of electron microscopes, are unstable, which will lead to an under-estimation of the true spatial resolution of the microscope.

The long recording time shown possible using the STEHM enables very low dose imaging necessary for electron beam sensitive specimens. The STEHM's measured stability may be dependent more on the specimen instabilities, which produce decoherence of the electron beams, rather than the microscope instabilities.

1. Herring, R.A., David Hoyle, Yoshifumi Taniguchi and Max Haider, "The Ultra-Stable Scanning Transmission Electron Holography Microscope" Microscopy \& Microanalysis, (Indiannapolis, August 4-8) 19 (Suppl 2) (2013) 302/303. 
2. Herring R A, Saitoh K, N, Tanaka N, and Tanji T (2010) Coherent Electron Interference from Amorphous TEM Specimens. J. Electron Microscopy 59: 321.

3. Herring R A, Saitoh K, N, Tanji T, and Tanaka N (2012) Electron interference from an amorphous thin film on a crystal transmission electron microscopy specimen. J. Electron Microscopy 61: 17.

4. These proceedings.

Funding from NSERC, CFI and BCKDF are gratefully acknowledged.

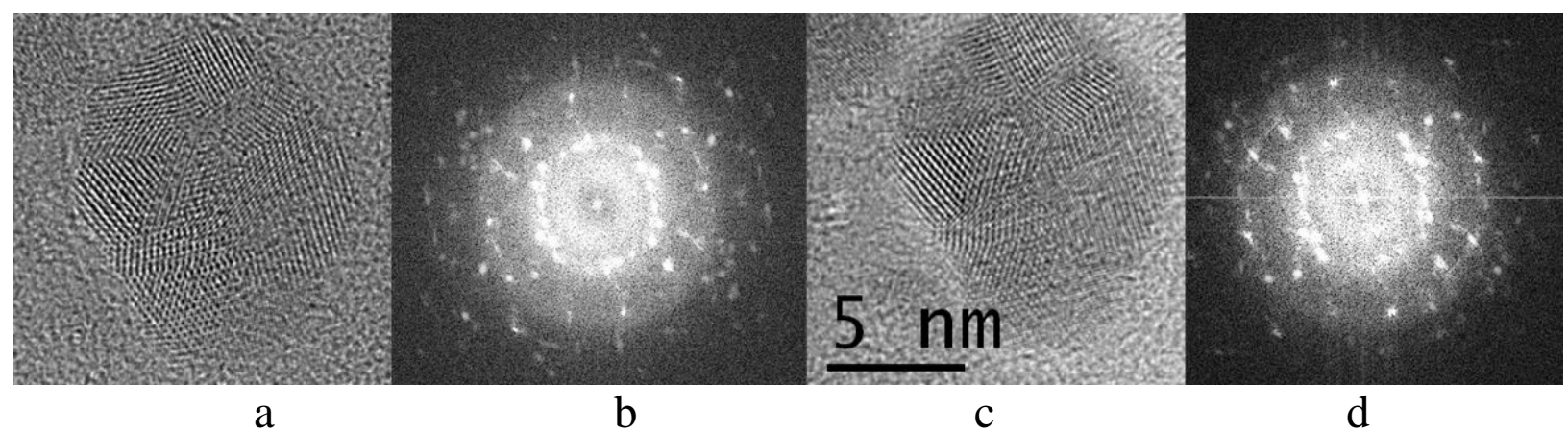

Figure 1 - TEM images and their corresponding Fourier transforms of gold crystals on an amorphous carbon substrate taken for $2 \mathrm{~s}$ in a and b and for $120 \mathrm{~s}$ in $\mathrm{c}$ and d. Contrast differences are due to specimen instabilities. Recording lattice images at $120 \mathrm{~s}$ enables high-resolution low dose imaging.
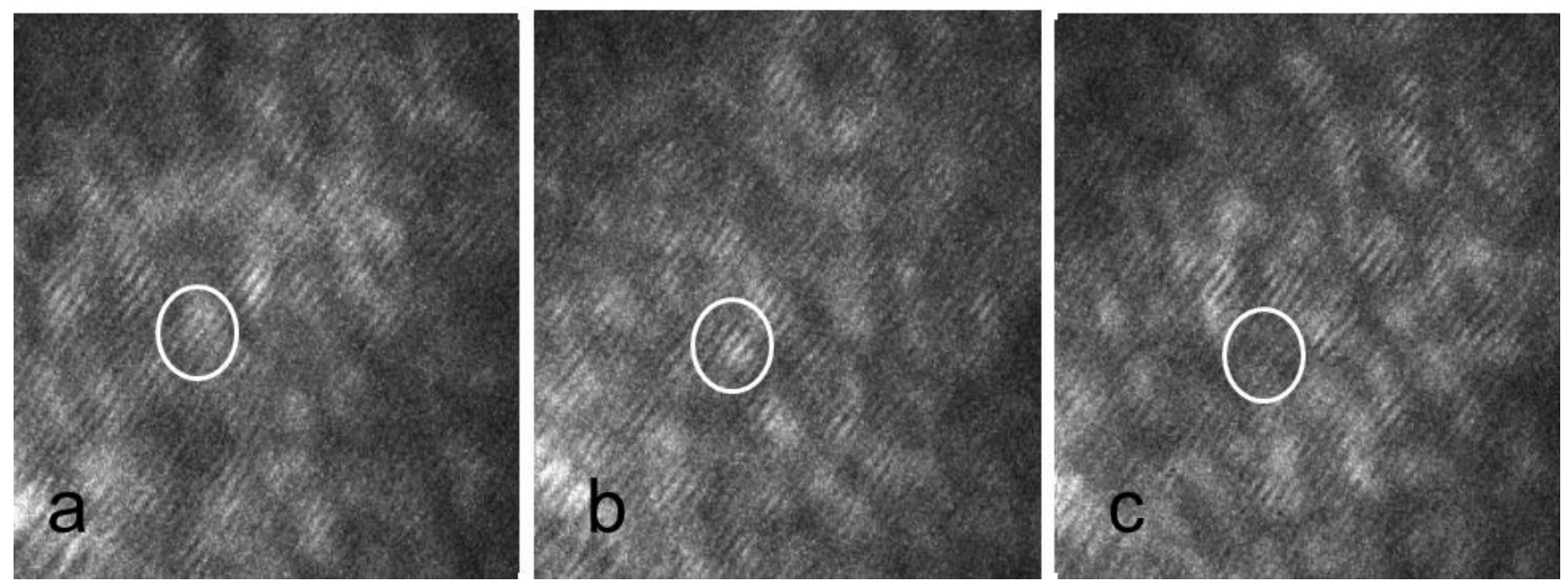

Figure 2 - Decoherence of interference fringes within the amorphous $\mathrm{C}$ intensities due to specimen instabilities. Images are recorded within a few seconds of each other from panels $a, b$, c. White circle is a reference location. 